\title{
Estimated effects of implementing an open access policy for grantees at a private foundation
}

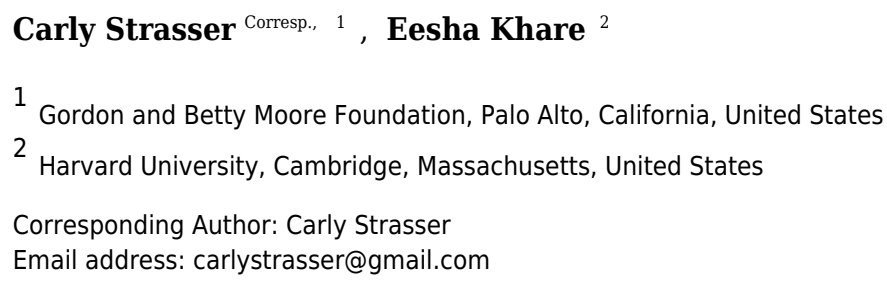

Background: The Gordon and Betty Moore Foundation (GBMF) was interested in understanding the potential effects of requiring that grantees publish their peer-reviewed research in open access journals. Methods: We collected data on more than 2000 publications in over 500 journals that were generated by GBMF grantees since 2001 . We then examined the journal policies to establish how two possible open access policies might have affected grantee publishing habits. Results: We found that $99.3 \%$ of the articles published by grantees would have complied with a policy that requires open access within 12 months of publication. We also estimated the maximum annual costs to GBMF for covering fees associated with "gold open access" to be between $\$ 400,000$ and $\$ 2,600,000$ annually. Discussion: Based in part on this study, GBMF has implemented a new open access policy that requires grantees make peer-reviewed publications fully available within 12 months. 


\section{Estimated effects of implementing an open access policy for grantees} 2 at a private foundation

3

4 Carly Strasser ${ }^{1}$ and Eesha Khare ${ }^{2}$

5

$6{ }^{1}$ Gordon and Betty Moore Foundation, Palo Alto, California, USA

7 2Harvard University, Cambridge, Massachusetts, USA

8

9 Corresponding author:

10 Carly Strasser ${ }^{1}$

11 Email address: carlystrasser@gmail.com 


\section{Abstract}

13 Background: The Gordon and Betty Moore Foundation (GBMF) was interested in

14 understanding the potential effects of requiring that grantees publish their peer-reviewed research

15 in open access journals. Methods: We collected data on more than 2000 publications in over 500

16 journals that were generated by GBMF grantees since 2001. We then examined the journal

17 policies to establish how two possible open access policies might have affected grantee

\section{Introduction} publishing habits. Results: We found that $99.3 \%$ of the articles published by grantees would have complied with a policy that requires open access within 12 months of publication. We also estimated the maximum annual costs to GBMF for covering fees associated with "gold open access" to be between $\$ 400,000$ and $\$ 2,600,000$ annually. Discussion: Based in part on this study, GBMF has implemented a new open access policy that requires grantees make peerreviewed publications fully available within 12 months.

In recent years, evidence of the benefits of open access (OA) for stakeholders in scientific research has been mounting. OA benefits researchers by increasing visibility of their research (Wang et al. 2015) and increasing citations counts (Gargouri et al. 2010). OA also helps build research capacity in developing countries (Chan et al. 2005), and decreases financial pressure on academic and research libraries (McGuian and Russell 2008). Funders are interested in promoting OA to increase visibility of the work that they fund, thereby increasing its potential impact. Increased visibility of research also reduces duplicative efforts and therefore duplicative investment. Further, OA research is available not just to academic researchers, but also to industry and the general public, thereby increasing reach and potentially resulting in a higher return on investment (Tennant 2016). Because of the growing body of evidence around OA benefits, many funders are establishing OA policies that mandate unrestricted online access to articles published in scholarly journals. Funders who have implemented policies recently include the Bill and Melinda Gates Foundation, the European Commission, US federal funders including National Science Foundation and the National Institutes of Health, and the Wellcome Trust.

It is generally agreed that "open access" refers to online research outputs that are free of all restrictions on access (e.g., fees or login requirements) and free of many restrictions on use (e.g., 
41 certain copyright and license restrictions) (Suber 2012). Here we consider the two main ways

42 that authors can make their work OA, gold and green (Harnad et al. 2008). In gold OA, the

43

44

45

46

47

48

49

50

51

52

53

54

55

56

57 58

59

60

61

62

63

64

65

66

67

68

69

70 content is freely accessible at the time of publication. Some journals require article processing charges (APCs) to cover the cost of making content freely available. Green OA refers to author "self-archiving", i.e., the content is posted online in an institutional or subject repository, or to a personal website. This method requires compliance with publisher or journal policies on selfarchiving, and are often subject to embargoes (i.e., a period during which an OA version of the article cannot be made public via self-archiving).

There is evidence that researchers are starting to consider openness in journal selection (Priem 2013), however this shift has yet to result in most articles being OA (Piwowar et al. 2017). Anecdotal evidence suggests most authors may not select journals based on their level of openness or their policies around self-archiving, instead choosing the most relevant, highest impact journal for their field. This is further complicated by the fact that some of the journals with highest impact factor are closed and do not allow for green or gold OA options. Funder policies around publishing OA would require that researchers more carefully consider their choice of journal to ensure compliance.

In an effort to increase access to the research results it funds, in 2016 the Gordon and Betty Moore Foundation (GBMF) began considering the implementation of an open access policy for all publications produced by its grantees. GBMF funds research in basic science, environmental conservation, patient care improvements, and preservation of the San Francisco Bay Area. With an annual budget of approximately $\$ 300$ million, GBMF has awarded approximately 2,400 grants since its founding in 2001. Although the foundation's existing Data Sharing and Intellectual Property Policy generally favors public access to grant outputs, at the time of this study it did not mandate open access.

Prior to implementing a new policy mandating open access, GBMF was interested in better understanding its effects on both grantees and the foundation's spending. GBMF was particularly interested in ensuring any policy did not infringe on the independence and expertise of grantees. That is, GBMF operates on the principle that grantees are most attuned to the needs of their discipline, and most likely to understand how best to disseminate their research to ensure high impact. GBMF carefully considers any policies that might restrict the normal activities of its 
71 grantees regarding their career path, scholarship, or results dissemination. An OA policy

72 prohibits grantees from publishing in the journal they believe is best would not be ideal since it

73 assumes we are better informed as to how the grantee should conduct their research endeavors.

74 The current policy of the foundation encourages openness, but does not weigh in on journal 75 choice.

76 We explored the potential costs of a policy change for GBMF that mandated OA, and how this 77 policy may affect journal choice for various types of GBMF grantees. We were most interested 78 in (1) whether grantees would be restricted from publishing in journals they have previously 79 chosen, and (2) the financial ramifications of a policy advocating for and funding gold OA when 80 available. Journals chosen by grantees in the past were not influenced by any GBMF 81 requirements or policies; we may assume that the list of journals we report here may be 82 influenced in the future by such policies. Since this work was completed, GBMF has 83 implemented a new open access policy that requires all peer-reviewed publications be openly 84 available within 12 months of publishing.

85 Although many funding organizations have mandated OA in some form, we are not aware of any 86 that have published results from internal analyses on the effects of such mandates. Kiley (2014) 87 provided data on Wellcome Trust's spending on APCs for grantees, however this was not 88 analyzed formally in a publicly available article. The dearth of available information about 89 effects of OA from a funder perspective is at least in part due to eclectic methods of grantee 90 reporting used by different funders. Grantee reports are not often machine readable or easily 91 analyzed in bulk. Some funders employ external firms to collect data on grantees' activities and 92 publications, but these are primarily used for internal decision-making and are not made public.

93 Other funders interested in pursuing mandates may benefit from understanding the results of our 94 study, and the transparency of publishing our analyses is evidence of our commitment to 95 promoting open research. Other potential audiences include policy makers and scholarly 96 communication researchers.

\section{Methods}

98 To explore potential impacts of an open access policy at GBMF, we analyzed 2650 publications 99 produced by GBMF grantees between 2001 and 2017 (Strasser and Khare 2017a). This is not a 
100 complete list of publications since the foundation does not yet have a standardized way of 101 collecting grantee outputs. The dataset includes publication data obtained from Science Program 102 grantee reports, as well as publication data from Crossref's Funding Data Search service 103 (Crossref 2017). Publications were deduplicated, grouped by journal title, and journal policy 104 metadata was added by searching the SHERPA-RoMEO database (Jisc 2017) of publisher 105 policies on self-archiving and open access.

106 Based on information found on journal websites and SHERPA-RoMEO, we classified each 107 journal as either open access, closed access, or hybrid. OA journals provide access to all content 108 immediately online (gold OA). Closed access journals restrict access to their content by requiring 109 that readers log into their website, usually to verify access to an institutional subscription. Hybrid 110 journals are closed access journals that provide authors with the option to opt into OA by paying 111 a fee (gold OA); only those articles that are designated OA are available publicly online. We also 112 determined whether the journal allowed authors to archive post-prints (peer-reviewed versions of 113 articles, but not necessarily with the publisher's formatting) - known as green OA, and the 114 length of the embargo period for archiving post-prints. The number of articles per journal is also 115 included in this article's corresponding dataset (Strasser and Khare 2017b).

116 The potential costs to GBMF for gold OA publishing were of interest, regardless of the policy 117 chosen. This would be particularly relevant if GBMF planned to provide financial support for 118 grantees publishing gold OA in journals with APCs. We therefore calculated an annual estimate 119 of maximum costs for gold OA for 2009-2016. We chose to analyze only a subset of years since 120 our dataset is limited and we have reason to assume the number of publications in years prior to 1212009 are vastly undercounted (less than 100 publications were found in years prior to 2009 in our 122 data collection). We multiplied the number of articles published in a year by 0.89 , which is the 123 percent of articles from our dataset that were published in hybrid or open journals and could 124 therefore be made gold OA. We then estimated the maximum annual cost for making these 125 articles OA by multiplying the value by $\$ 3000$, the high end of the APC estimate from West et 126 al. (2014). Others report lower average APC costs (e.g., Romeu 2014), however we chose the 127 highest estimate to capture the fact that this was intended to estimate the potential maximum cost 128 of OA. 
129 Our study is limited by the availability of consistent, complete data for GBMF grantees. Annual 130 reports are not collected in consistent, machine readable ways that lend themselves to bulk 131 analysis. We also have no data on APCs already paid for using GBMF funds out of grantee

132 budgets. Efforts are underway at GBMF to develop systems for consistent, reliable collection of 133 grantee publication data, however these were not implemented in time to benefit this study.

\section{Results}

135 Our data collection yielded a list of 573 journals used by grantees, in which 2650 articles were 136 published. We were first interested in the percentage of journals chosen by our grantees that are 137 hybrid, open, or closed. We calculated percentages both by journal and by article to ensure that 138 we captured potential effects on authors, however the numbers were quite similar (Figure 1). 139 GBMF grantees tend to publish in hybrid journals (74\% of journals; $72 \%$ of articles), with open 140 (16\% of journals; $17 \%$ of articles) and closed (10\% of journals; $11 \%$ of articles) journals less 141 represented. Although we have no information on the percentage of those articles published in 142 hybrid journals that were published gold OA, there is evidence that without funder mandates, the 143 percentage of articles that are published OA is low (Tennant et al. 2016). 


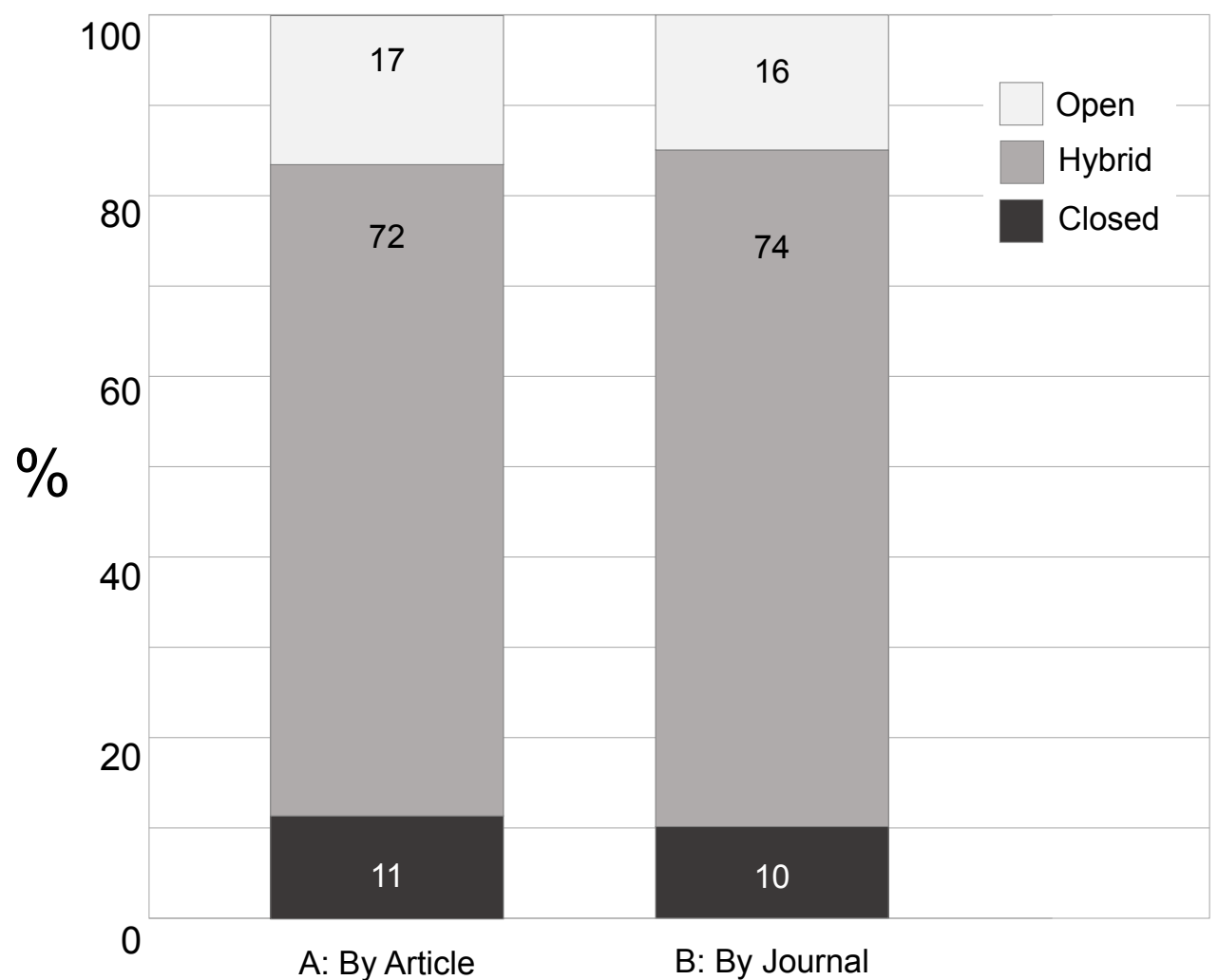

145

146

147

148

149

150

151

152

153

154

155

156

157

158

159

160

Figure 1. Breakdown of journal type (open, hybrid, or closed) used by GBMF grantees for the 2650 articles (A) published in 573 journals (B). Data: Strasser and Khare 2017a, 2017b

We were also interested in whether a grantee's journal choice would be impacted by two possible OA policies being considered. Policy A would require OA within 12 months of publication, either via the green or gold route. Grantees could comply by either publishing in open journals, by publishing OA in a hybrid journal (gold OA), or by publishing non-OA and self-archiving within 12 months (green OA). This policy would preclude grantees from publishing in journals that are closed and restrict self-archiving for longer than 12 months. Policy B would require immediate access at the time of publication (gold OA). Grantees could comply by either publishing in open journals, or by publishing OA in a hybrid journal. In some cases, journals may allow immediate access at the time of publication (no embargoes on post-prints); this route of OA would also be acceptable (but is much less frequent in journals used by grantees). Policy B excludes journals that are closed and/or restrict self-archiving at the time of publication. 
161 Based on these proposed policies, we calculated that the percentage of articles in our dataset that 162 would have been compatible with Policy A is 99.3. The articles that would not have been 163 compatible with Policy A were exclusively from the family of Annual Reviews. These journals 164 are not typical publications since they do not publish original research and are instead reviews of 165 work in various disciplines.

166 For Policy B, 8.3\% of articles in our dataset would not have been compatible. These are 167 primarily high impact journals with no gold OA option that restrict post-print archiving for some 168 period of time. Journals that do not comply include the family of Nature journals (except Nature 169 Communications) and Science, published by AAAS (American Association for the Advancement 170 of Science).

171 The estimated maximum cost for implementing Policy B (i.e., gold OA for all publications) 172 ranged from $\$ 401,835$ in 2008 to $\$ 2,538,725$ in 2015. (Figure 2). This large range is a direct 173 consequence of the variable amount of data available on articles across years. For example, we 174 identified 814 publications in 2015 (compared to 328 and 501 in the years before and after, 175 respectively). In 2015, the newly funded Data-Driven Discovery Initiative

176 (https://www.moore.org/initiative-strategy-detail?initiativeId=data-driven-discovery) in the 177 Science Program began systematically collecting data from its grantees about publications, 178 resulting in the large spike. Such inconsistencies in the data are unavoidable given the absence of 179 methods for collecting data systematically. 
3

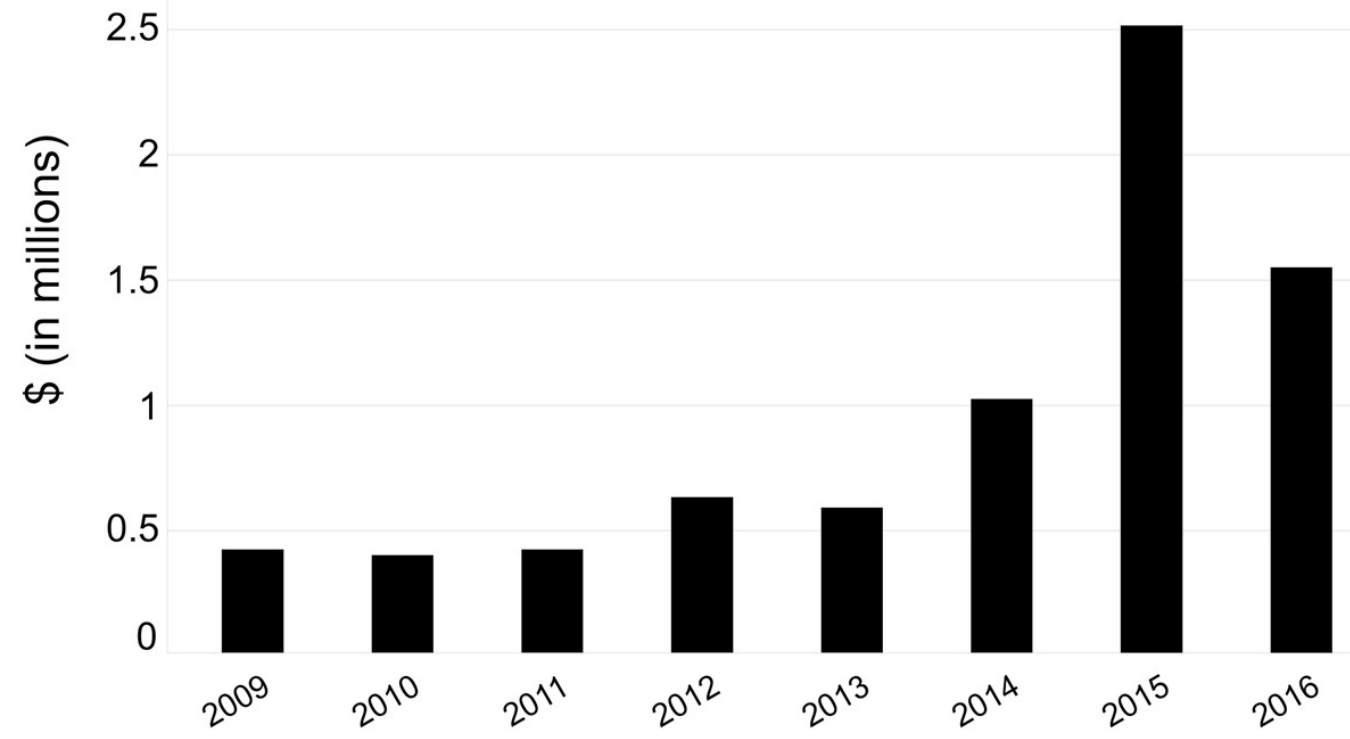

180

181

182

183

184

Figure 2. Estimated maximum annual costs for OA fees associated with GBMF grantee publications Data: Strasser and Khare 2017a, $2017 b$.

185

186

187

188

189

190

191

192

193

194

195

196

197

198

\section{Discussion}

Based on the data reported here, the OA policies considered by GBMF would not have substantially altered the journals in which the grantees published their work. This is particularly true for proposed Policy A (green or gold OA within 12 months). Only $0.7 \%$ of articles published by grantees would not have complied with this policy. If the gold OA policy option were enacted (Policy B), some journals that restrict access to all their content for up to 12 months after publication would not be permissible. Only $8.3 \%$ of articles published by grantees in our dataset would not have complied with this policy.

Implementing an OA policy at a private foundation has potential implications beyond ensuring grantees make their work open access. It also serves to advocate for a position of openness in research outputs. Such a policy might encourage grantees to select journals with more open policies, or may encourage them to use grant funds to choose gold OA (even without a mandate). Of course, requirements imposed by funders for OA will inevitably result in more accessible work. A study by Xia (2012) found that hundreds of policies have been proposed and adopted at 
199 various organizational levels and many of them have resulted in increased self-archiving (i.e., 200 green OA).

201 The potential maximum financial ramifications of paying APCs for all articles were estimated to 202 be between $\$ 400,000$ and $\$ 2,600,000$ per year. There are several unknown factors that might 203 influence what amount within this range is likely to be correct for GBMF, most critically how 204 many publications are generated each year by grantees. Our estimates of number of publications 205 per year (and therefore estimated maximum APC costs) rely on either the grantee self-reporting 206 to the foundation, or the grantee including GBMF in manuscript acknowledgements that can be 207 harvested by Crossref. Our range of costs is therefore likely an underestimate for earlier years 208 that have lower publication counts due to the increased difficulty in tracking down data from 209 those years. Potential overestimates at the higher end of the range would result from assuming 210 GBMF would incur costs for all publications generated by grantees.

211 The estimated maximum cost for implementing Policy B (i.e., gold OA for all publications) is 212 the result of several simplifications. First, we account for any potential institutional 213 memberships, wherein publishers charge institutions a fixed annual fee which covers some 214 percentage of article-processing fees for the institution for the year. These memberships may 215 result in discounts, which may result in significantly reducing costs for OA (Smith et al. 2017). 216 Second, our calculation also does not correct for the fact that many OA journals do not charge 217 APCs (see http://www.doaj.org), and therefore the maximum cost for GBMF to fund gold OA 218 would be lower.

219 There are other considerations in OA policy implementation that were not the subject of this 220 research. For instance, some funders have chosen to cease funding APCs for OA articles in 221 hybrid journals, opting instead to use funds to cover costs for fully OA journals only. This 222 strategy is used by the German Research Foundation and the Norwegian Research Council, and 223 is a response to high prices and poor service, and some journals' tendency to charge for articles 224 where APCs have been paid due to internal error (Butler 2016).

225

226 GBMF announced a new Open Access Policy in 2017 (Moore Foundation 2017). The new policy 227 states that 
228

229

230

231

232

233

234

235

236

237

238

239

240

241

242

243

244

245

246

247

248

249

250

251

252

253

254

255

256
The foundation requires that a final (post-print) version of all peer-reviewed articles produced as a result of research supported, either in entirety or in part, by the foundation's funding, be made publicly and freely available (open access, or OA) within 12 months of publication. Grantees can accomplish this either by publishing the article $O A$, by ensuring that the publisher will make the content $O A$ within 12 months, or by depositing a post-print version of the manuscript in an OA repository within 12 months.

This is equivalent to Policy A described above. The decision to implement Policy A as opposed to something more prescriptive, such as Policy B, was based in part by GBMF's policy to allow grantees to make decisions about their research and its dissemination based on their expertise, rather than our policies. GBMF funds a wide breadth of research, and not all grantees will be impacted by an OA policy in the same way. The Environmental Conservation Program and the Science Program both are likely to have grantees that produce peer-reviewed publications, and are likely to be impacted by the OA policy. However even within these two Programs, there are a diversity of disciplines represented that may require different amounts of behavioral changes in publishing habits to comply with the policy. The new policy at GBMF will serve to "level up" the different groups, ensuring that the public can access all peer-reviewed publications generated by its grantees.

Another factor in choosing Policy A over Policy B was the potential ramifications of encouraging gold OA without considering how fees will be paid. Some funders have separate budgets for APCs, while others require that this cost be included in the grant budget. GBMF was interested in a more conservative approach, which will result in time to gather data and concerns about implementing a policy that requires gold OA.

GBMF plans to revisit the efficacy and impact over time for the newly implemented policy. There are several variables that can be altered to potentially strengthen the OA policy. These may include (1) restricting embargoes on archiving OA versions to six months (compared to 12 months); (2) requiring CC-BY licenses for all publications; (3) setting aside funds for covering grantees' OA fees (independent of their grant funds); (4) mandating particular repositories for OA archiving; or (5) expanding the policy to include outputs other than peer-reviewed journals (e.g., data, software, books, etc.). 


\section{Acknowledgements}

258 The authors would like to thank N. Caulk, J. Lin, and A. Jones for helpful comments on the 259 manuscript. K. Canesi provided data. C. Mentzel and N. Caulk provided helpful insights 260 throughout the project. We are especially grateful for the two reviewers (M. Winker and 261 anonymous) who gave helpful feedback that resulted in a much better manuscript.

\section{References}

263 Butler, D. 2016. Dutch lead European push to flip journals to open access. Nature 529.

264 http://doi.org/10.1038/529013a.

265

266

267

Chan, L., Kirsop, B., Arunachalam, S. 2005. Open access archiving: the fast track to building

268 research capacity in developing countries. Science and Development Network.

269

270

271 http://hdl.handle.net/1807/4415

Crossref 2017. Fundref metadata search. http://search.crossref.org/funding (accessed 15 January 2017)

272

273

Gargouri, Y., Hajjem, C., Larivière, V., Gingras, Y., Carr, L., Brody, T., Harnad, S. 2010. Self-

274 Selected or mandated, open access increases citation impact for higher quality research. PLOS

275 ONE 5 (10), e13636+. http://doi.org/10.1371/journal.pone.0013636

276

277

Harnad, S., Brody, T., Vallieres, F., Carr, L., Hitchcock, S., Gingras, Y., Oppenheim, C., Hajjem, 278 C., Hilf, E. 2008. The access/impact problem and the green and gold roads to open access: An

279

280

281

282

283

284 update. Serials Review 34 (1), 36-40. http://doi.org/10.1080/00987913.2008.10765150.

Jahn, N., Tullney, M. 2016. A study of institutional spending on open access publication fees in germany. PeerJ 4. https://peerj.com/articles/2323/

286

287

Jisc 2017. SHERPA-RoMEO database of publisher copyright policies and self-archiving. http://www.sherpa.ac.uk/romeo/ (accessed 1 August 2016 - 1 April 2017).

Kiley, R. 2014. Wellcome Trust APC spend 2012-13: data file. figshare.

289 https://doi.org/10.6084/m9.figshare.963054.v1. Retrieved 15:11, Aug 09, 2017 (GMT)

290

291

McGuigan, G., Russell, R. D. 2008. The business of academic publishing. Electronic Journal of

292 http://southernlibrarianship.icaap.org/content/v09n03/mcguigang01.html (accessed 15 March 293 2017) 
294

295

296

297

298

299

300

301

302

303

304

305

306

307

308

309

310

311

312

313

314

315

316

317

318

319

320

321

322

323

324

325

326

327

328

329

330

331

332
Moore Foundation 2017. Moore Foundation rolls out new open access policy.

https://www.moore.org/article-detail?newsUrlName=moore-foundation-rolls-out-new-openaccess-policy\&tagToFilterBy=ab660061-a10f-68a5-8452-ff00002785c8 (accessed 18 April 2017)

Piwowar, H. Priem, J., Larivière,V., Alperin, J.P., Matthias, L., Norlander, B., Farley, A., West, J., Haustein, S. 2017. The State of OA: A large-scale analysis of the prevalence and impact of Open Access articles. PeerJ preprint 5. e3119v1. https://doi.org/10.7287/peerj.preprints.3119v1.

Priem, J. 2013. Scholarship: Beyond the paper. Nature 495 (7442), 437-440.

http://doi.org/10.1038/495437a

Romeu, C.,Gentil-Beccot, A., Kohls, A., Mansuy, A., Mele, S., Vesper, M. 2014. The SCOAP3 initiative and the Open Access Article-Processing-Charge market: global partnership and competition improve value in the dissemination of science. CERN Report Number CERNOPEN-2014-037. 15 pp. http://doi.org/10.2314/CERN/C26P.W9DT.

Smith, A., Sartori, A., \& Office of Scholarly Communication. 2017. Report presented to Research Councils UK for article processing charges managed by the University of Cambridge, 2016-2017 [Dataset]. https://doi.org/10.17863/CAM.9809

Strasser, C., Khare, E. 2017a. Moore grantee list of articles. Zenodo.

http://doi.org/10.5281/zenodo.841794.

Strasser, C., Khare, E. 2017b. Moore grantee publication data. Zenodo.

http://doi.org/10.5281/zenodo.555947.

Suber, P. 2012. Open Access. The MIT Press Essential Knowledge Series, MIT Press. 255p.

Available at https://mitpress.mit.edu/books/open-access.

Tennant, J. P., Waldner, F., Jacques, D. C., Masuzzo, P., Collister, L. B., Hartgerink, C. 2016. The academic, economic and societal impacts of open access: an evidence-based review. F1000Research 5, 632+. http://doi.org/10.12688/f1000research.8460.3

Wang X., Liu C., Mao W., Fang, Z.. 2015. The open access advantage considering citation, article usage and social media attention. Scientometrics 103 (2), 555-564.

http://doi.org/10.1007/s11192-015-1589-3. 
333 West, J.D., Bergstrom, T., Bergstrom, C.T. 2014. Cost effectiveness of open access publications. 334 Economic Inquiry 52 (4), 1315-1321. http://doi.org/10.1111/ecin.12117.

335

336 Xia, J., Gilchrist, S. B., Smith, N. X., Kingery, J. A., Radecki, J. R., Wilhelm, M. L., Harrison, 337 K. C., Ashby, M. L., Mahn, A. J. 2012. A Review of Open Access Self-Archiving Mandate 338 Policies. Libraries and the Academy 12 (1). 85-102. http://doi.org/10.1353/pla.2012.0000.

339

340

341

342

343

344 